\title{
Ausbildungsaufgaben als Instrument der universitären fachdidaktischen Ausbildung in der Sprachbildung
}

Prof. Josef Leisen

Staatliches Studienseminar Koblenz

Institut für Physik

Universität Mainz

\section{Einleitung}

Sprachbildung ist in allen Fächern, in allen Schulstufen und Schulformen zu einem zentralen Thema geworden. Das Bewusstsein für einen sprachbildenden und sprachsensiblen Sach-Fachunterricht ist deutlich gestiegen. Die LehrerInnenschaft nimmt im täglichen Unterricht vor Ort wahr, dass der Sach-Fachunterricht durch wachsende Heterogenität beim Sprechen, Lesen und Schreiben anstrengender und mutmaßlich weniger lernwirksam ist, dass es an lexikalischer Kompetenz fehlt und dass die Sprachprobleme wachsen. (Die in Deutschland erschienenen Studien DESI-Studie 2006, Sprachliche Integration von Migranten in Deutschland 2008, BISS-Expertise 2012, Kinder-Migrationsreport 2013 reagieren einerseits differenzierend auf die Wahrnehmungen der Lehrkräfte und fokussieren andererseits Förder- und Unterstützungsmaßnahmen.) Die LehrerInnenschaft ahnt und erkennt zunehmend, dass Fachlernen nicht ohne Sprachlernen funktioniert, dass beides in den Sach-Fachunterricht gehört. Aus dem Unterrichtsalltag heraus entsteht unter praktizierenden Lehrkräften somit ein enormer Fortbildungsbedarf. Die Not ist groß und die Zeit drängt.

Angehende Lehrkräfte sollten bereits in der universitären Ausbildung ein Grundwissen im Bereich „Sprachbildung - Spracherwerb - Sprachförderung“ erwerben, für die sprachlichen Belange in den Fächern sensibilisiert werden und wünschenswerterweise bereits erste unterrichtspraktische Erfahrungen sammeln 
können. FachdidaktikerInnen in den Universitäten sind hier gleichermaßen gefordert wie AusbilderInnen in den Studienseminaren und FortbildnerInnen in den Fortbildungsinstitutionen. Wer aber bildet die Personen in der Aus- und Fortbildung aus? Die Frage läuft auf das Henne-Ei-Prinzip hinaus, wobei der Grundsatz gilt, dass es zum Kompetenzprofil dieser Personengruppe gehört, sich überwiegend selbst auszubilden und durch die Unterstützung über Netzwerke und Expertisen von außen kompetent zu machen. Die Aufgabe ist gleichsam eine doppelte: sich selbst zu qualifizieren und parallel mitlaufend bzw. mit zeitlicher Versetzung die Studierenden auszubilden. Die in der Ausbildung tätigen Personen müssen die in Abb. 1 dargestellten drei Ebenen im Blick haben und Verknüpfungen herstellen.

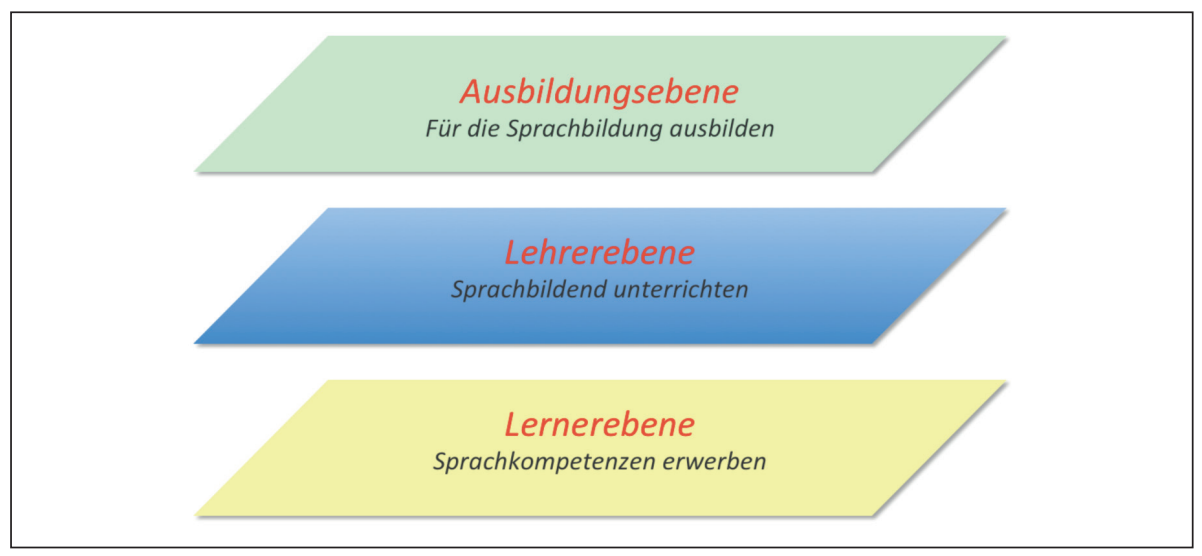

Abb. 1: Sprachbildung und Sprachlernen im Fach auf drei Ebenen (eigene Darstellung J. L.)

\section{Ausbildungsinhalte für den sprachsensiblen Sach-Fachunterricht}

Ohne an dieser Stelle ein Curriculum zu entfalten, werden in der Folge hinsichtlich der genannten drei Ebenen lediglich inhaltliche Elemente skizziert, die aus Sicht des Autors Teil der Ausbildung sein sollten. 
- Wissen über Sprache und Sprachbildung: Wie wird in meinem Fach kommuniziert?

Sprache(n) im Fach, Darstellungsformen, morphologische und syntaktische Besonderheiten, BICS und CALP, Schriftlichkeit und Mündlichkeit, Sprachbildung, ...

- Wissen über Spracherwerb und Sprachlernen: Wie erwerben und lernen LernerInnen die Sprache im Fach?

Spracherwerb und Zweitspracherwerb, Submersion und Immersion, Sprachlernen und Kognition, Fachlernen und Sprachlernen, ...

- Wissen über Sprachkompetenzen: Wie sprechen, lesen, schreiben LernerInnen in meinem Fach?

Sprachkompetenzen und sprachliche Fertigkeiten, Sprachprobleme, Sprachdiagnostik, ...

- Wissen über Sprachlernprozesse und Sprachförderung: Wie gestalte ich Sprachlernprozesse und wie fördere ich die LernerInnen beim Spracherwerb im Fach?

sprachsensibler Sach-Fachunterricht, sprachliche Kompetenzentwicklung, sprachliche Fördermethodik, sprachliche Handlungskompetenz im Fach, Methoden-Werkzeuge, individuelle Sprachförderung, Umgang mit sprachlicher Heterogenität, Sprachsensibilität bei Aufgabenstellungen, Scaffolding, Umgang mit sprachlichen Fehlern, Bewertung von Sprache im Fach, ...

Dadurch, dass es sich hierbei um ein Querschnittsthema handelt, greift eine verdünnte grundständige Fachausbildung zu kurz. Die Thematiken sollten entsprechend umfassender implementiert werden. Denn wie bei allen Querschnittsthemen lauert ansonsten die Gefahr, dass niemand das Anliegen als originär eigen ansieht und das Thema somit allen, aber letztlich niemandem gehört und es zwischen den Fachbereichen, Instituten und Institutionen evaporiert.

Das folgende didaktische Dreieck zeigt die Sprachbildung im Schnittpunkt von drei Didaktiken: der Sach-Fachdidaktik, der Sprachlerndidaktik im Fach und der Fremdsprachendidaktik. 


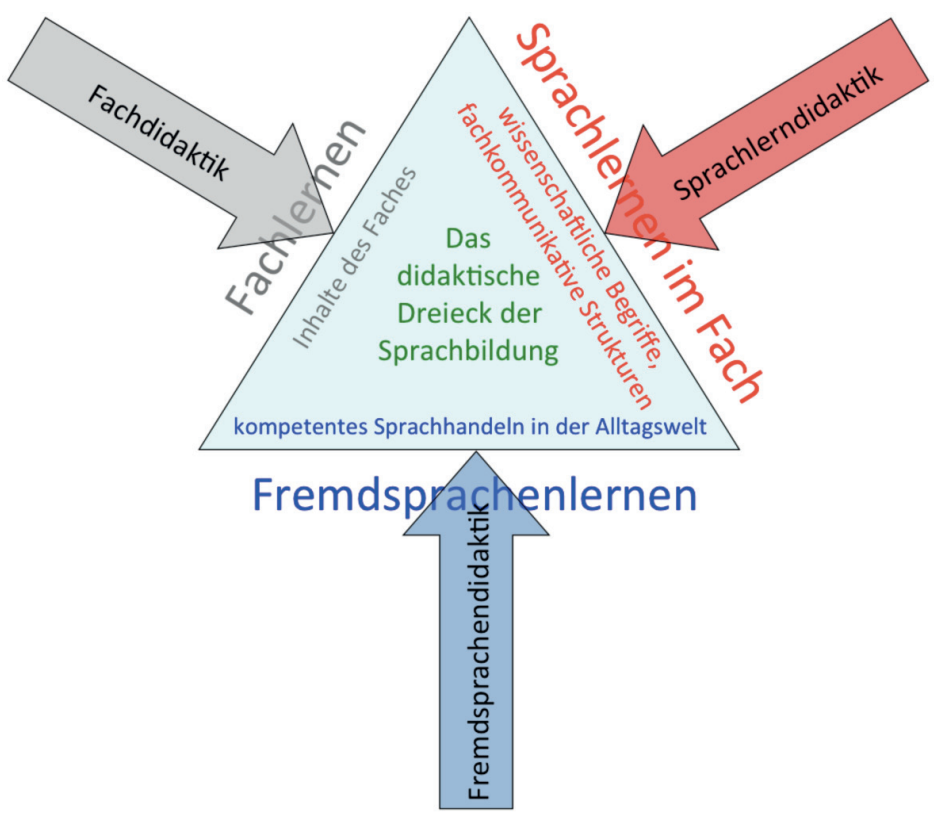

Abb. 2: Das didaktische Dreieck der Sprachbildung (eigene Darstellung J. L.)

Der sprachsensible Sach-Fachunterricht umfasst zwei Themen, nämlich das Lernen im Fach (Fachlernen) und das Lernen der Sprache im Fach (Sprachlernen) unter Berücksichtigung von Prinzipien und Methoden des Fremdsprachenlernens. Eine Lehrkraft, die nur das Fachlernen sieht und das Sprachlernen im Fach als marginal und nebenherlaufend betrachtet, hat nicht die passende Auffassung für einen sprachsensiblen Sach-Fachunterricht. Sie wird den Konnex von Sprache und Fachkonzepten nicht richtig einschätzen und womöglich Verstehen dort zuschreiben, wo Fehlvorstellungen vorherrschen. Diese Lehrkraft wird die Sprache als ein wichtiges Diagnoseinstrument für fachliches Verstehen unterschätzen.

Die Merkmale eines sprachsensiblen Unterrichts sind leicht zu formulieren, wenngleich nicht immer einfach umzusetzen. Die Merkmale eines sprachsensiblen Unterrichts sind (vgl. Leisen 2013: 3f): 
- Fachlernen wird zum Sprachlernen genutzt.

- LernerInnen tauchen in ein sprachlich reichhaltiges Sprachbad ein (Immersion).

- LernerInnen werden sprachlich gezielt herausgefordert.

- Die Sprachprobleme der LernerInnen werden erkannt.

- Es wird diagnostiziert, wo und wie unterstützt werden kann.

- Sprachhilfen mit Methoden-Werkzeugen werden angeboten.

- Ansätze der Fremdsprachdidaktik (Vorentlastungen, Kontexte, Fehlerkorrektur, ...) werden genutzt.

Das sprachliche Thema des sprachsensiblen Sach-Fachunterrichts ist der angemessene Erwerb der Bildungssprache im jeweiligen Fach. Hier treffen nach Abb. 2 Fachlernen und Sprachlernen zusammen und können nicht voneinander getrennt werden, weder (sach-)fachdidaktisch noch sprachlerndidaktisch noch fremdsprachendidaktisch.

\section{Kompetenzen für den sprachsensiblen Sach-Fachunterricht}

Standards der Sprachbildung beschreiben die Kompetenzen (und deren Performanz), über die Lehrpersonen unbedingt verfügen müssen, damit sie unterrichtliche und sprachbildende Situationen (sprachliche Standardsituationen) erfolgreich und adäquat bewältigen. Standards sind notwendige Wissens- und Könnensbestände, durch die sich das professionelle Handeln in unterrichtlichen und schulischen Situationen von dem eines Laien unterscheidet. In Bezug auf die Sprachbildung werden folgende acht Standards aufgelistet (vgl. Feinschreibung und Erläuterungen in Leisen 2013).

- Standard 1: Über Wissen über Sprache, Sprachregister, Sprachmerkmale, Spracherwerb und Sprachbildung verfügen

- Standard 2: Über Wissen zur Sprachdiagnostik verfügen 
- Standard 3: Über didaktisches und methodisches Wissen zur Sprachbildung, zum Lesen und Schreiben verfügen

- Standard 4: Sprach-, Lese- und Schreibprozesse gestalten

- Standard 5: Mit sprachlichen Standardsituationen professionell umgehen

- Standard 6: Nachhaltige Sprachförderung durch Üben betreiben

- Standard 7: Sprachdiagnostische Methoden nutzen

- Standard 8: Sich in den Kompetenzen der Sprachbildung entwickeln

Kompetenzen umfassen Wissen, Können und Handeln ganz im Sinne der Referenzdefinition nach Weinert (Weinert 2001: 27f.; Klieme 2003: 15f.). In der Sprachbildung kompetente Personen müssen über Wissen, Fähigkeiten und Fertigkeiten verfügen und in Sprachbildungsprozessen professionell handeln können. Kompetenz zeigt sich in der Performanz, also im tatsächlichen Handeln.

Die Kompetenzen zur Sprachbildung müssen auf vier Ebenen erworben werden, wie in Abb. 3 dargestellt:

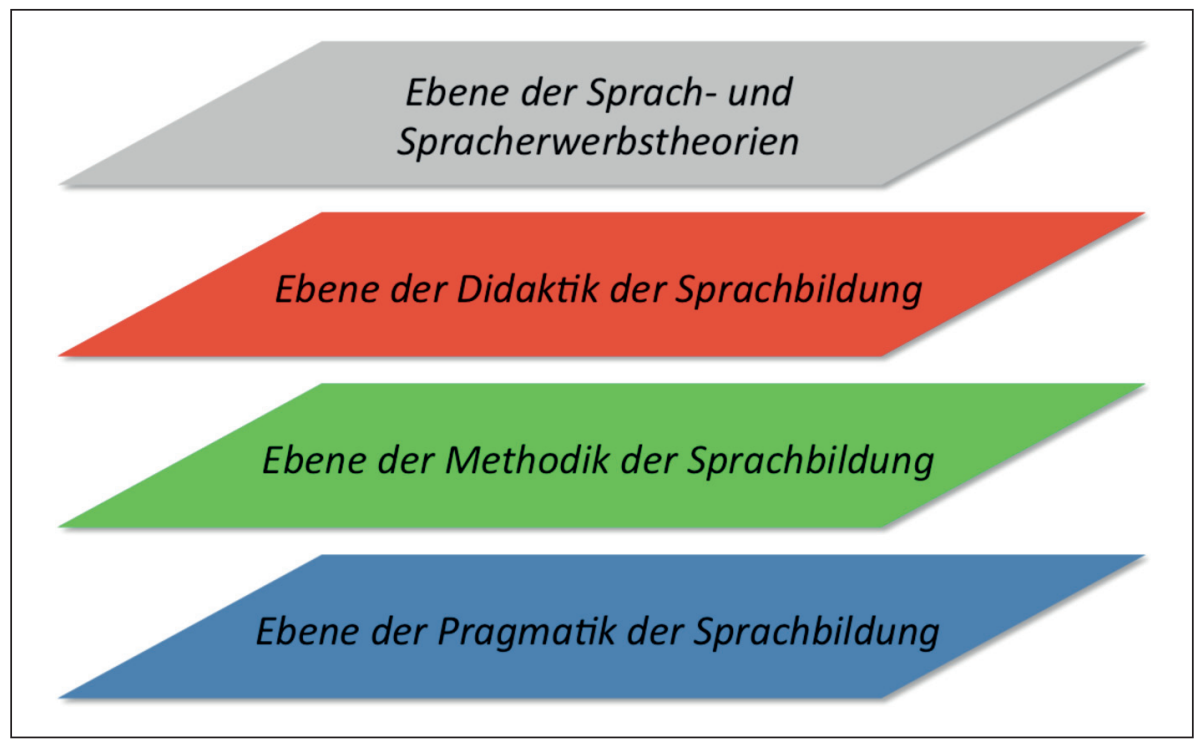

Abb. 3: Vier Ebenen des Kompetenzerwerbs zur Sprachbildung (eigene Darstellung J. L.) 
Praktizierende Lehrkräfte müssen rasch in den handelnden Umgang mit dem Wissen über Sprachbildung gebracht werden. Sie müssen dementsprechend von der Praxis aus zum Wissen hin fortgebildet werden, also theoriebegleitet und nicht theoriegeleitet. Aus der alltäglichen Praxis heraus müssen sie lernen, wie das Sprachlernen im Fach gefördert und Sprachlernprozesse gesteuert werden.

Studierenden hingegen fehlt das Erfahrungswissen. Sie können auch während des Studiums nicht in den handelnden Umgang mit dem Wissen gebracht werden, da ihnen die unterrichtlichen Möglichkeiten fehlen. Anzumerken ist, dass Praktika hier nicht ausreichen, zumal sich Studierende noch mit elementaren Lehrkompetenzen auseinandersetzen müssen (vgl. Jürgens 2016).

ReferendarInnen machen viele Erfahrungen mit Sprachproblemen im Unterricht. Sie erleben den Handlungsdruck tagtäglich und sind dankbar für eine entsprechende Ausbildung. Gleichwohl ist deren Zeitbudget, das sie für die Ausbildung im sprachsensiblen Sach-Fachunterricht aufwenden können, sehr beschränkt (vgl. Baumann \& Becker-Mrotzek 2014: 8; Lütke 2010; HumboldtUniversität zu Berlin 2015).

Personen, die in der Fortbildung und Schulentwicklung tätig sein werden, müssen auf mehreren Ebenen gleichzeitig tätig und kompetent sein. Sie müssen selbst über ein fundiertes Wissen zur Sprachbildung verfügen, sollten über eigene Unterrichtserfahrungen in der Sprachförderung verfügen und müssen zusätzlich über Wissen und Können im Bereich der Schulentwicklung, der Beratung, der Gestaltung und Moderation von Fortbildungsveranstaltungen verfügen.

\section{Plädoyer für eine aufgabenorientierte Ausbildung mit Ausbildungsaufgaben}

Kompetenzen für den sprachsensiblen Sach-Fachunterricht werden nicht gelehrt, sondern in Handlungen und in sprachbildenden Situationen an sprachdidaktischen Inhalten mit Ausbildungsaufgaben erworben.

Aufgaben und Aufgabenstellungen sind konstitutive Teile jeden Lernens, sei es im Unterricht oder in der beruflichen Ausbildung (Leisen 2011). In den Na-

Barbara Hinger (Hg.), Zweite „Tagung der Fachdidaktik“ 2015.

Sprachsensibler Sach-Fach-Unterricht - Sprachen im Sprachunterricht.

(C) 2016 innsbruck university press, ISBN 978-3-903122-51-2, DOI 10.15203/3122-51-2 
turwissenschaften haben Aufgaben im universitären Lehrveranstaltungstyp der Übungen eine lange Tradition. Nachweislich werden den Aufgaben dabei sehr hohe Lerneffekte zugesprochen. Folglich sollte dieses Instrument auf andere Fächer und auf die Fachdidaktik übertragen werden. Anzumerken ist, dass in der Fremdsprachendidaktik spätestens seit den 1980er Jahren der aufgabenorientierte Ansatz als wesentliche Erweiterung des kommunikativen Fremdsprachenunterrichts gilt (vgl. u.a. Ellis 2003).

Einer aufgabenorientierten Ausbildung liegen entsprechende Arbeitsmaterialien (Arbeitstexte, Materialien in elektronischer und nichtelektronischer Form, ...) zu Grunde. ,Aufgaben` sind in einem sehr allgemeinen Verständnis Aufforderungen an die Studierenden bzw. ReferendarInnen, in einer mehr oder weniger vorgezeichneten Bahn kognitiv aktiv zu werden.

\section{Eine Aufgabe im Studium besteht im Wesentlichen aus zwei Teilen:}

a) dem Setting:

- Rahmung der fachlichen bzw. fachdidaktischen Situation

- Darstellung des Kontextes

- Bereitstellung von Materialien (Texte, Bilder, Geräte, ...)

b) den Bearbeitungsaufträgen:

- Arbeitsaufträge in Form von Fragen oder Aufforderungen mittels Operatoren

- Angabe der Bearbeitungs- und Darstellungsmittel

- ggf. Hilfen und Hinweise zur Bearbeitung (Methoden, Ablauf, Zeit, Umfang, ...)

- Angaben zur Darstellung und Präsentation

- Angaben zur Bearbeitungsqualität und Bewertungskriterien

- oder dem Fehlen jeglicher Angaben

(vgl. Bund-Länder-Kommission 1997; Leisen 2005; Stäudel et al. 2012). 
Die Erfahrungen mit Beispielaufgaben, das sind Aufgaben mit Musterlösungen zum Selbststudium, zeigen eindeutig positive Effekte. Das gilt für den Bereich der Berufsausbildung ebenso wie für den schulischen Bereich (Kross \& Lind 2001). Hier sollen in der Folge Ausbildungsaufgaben definiert und Kriterien für gelungene Ausbildungsaufgaben aufgelistet werden.

\section{Definition der Ausbildungsaufgabe}

- Eine Ausbildungsaufgabe ist eine Aufgabe aus dem Lernraum zur beruflichen Kompetenzentwicklung (hier sprachsensibler Sach-Fachunterricht) der Studierenden und ReferendarInnen.

- Ausbildungsaufgaben enthalten Materialien aus der Praxis der Sprachbildung (Lehr- und Lernmaterialien, Lese- und Lernprodukte der LernerInnen, ...) und aus der Sprach- und Spracherwerbstheorie (theoretisches Hintergrundwissen).

- Von den Studierenden und ReferendarInnen werden auswertbare Lernprodukte (meistens Lernumgebungen) erstellt, an denen der Kompetenzstand rückgemeldet werden kann.

- Ausbildungsaufgaben realisieren sich in konkreten Vorhaben.

\section{Gute Ausbildungsaufgaben}

- aktivieren die ReferendarInnen zum selbstständigen Lernen.

- knüpfen am Vorwissen und der Wissensstruktur an und erweitern das Wissen.

- sind gestuft aufgebaut.

- sind in einen Ausbildungskontext und in ein Ausbildungscurriculum eingebettet.

- vernetzen mehrere berufliche Handlungssituationen.

- schaffen eine adäquate Atmosphäre des Lernens.

- orientieren sich am Kompetenzmodell der Standards.

- fördern das Könnensbewusstsein.

- zeigen den Lernzuwachs.

- verankern das neu gelernte im Wissens- und Könnensnetz. 


\section{Beispiele für Ausbildungsaufgaben}

\subsection{Beispiel 1: Volumenmessung eines beliebig geformten Körpers}

In den Vorstunden lernen die SchülerInnen der 5. Schulstufe im Mathematikunterricht die Volumenberechnung von Quadern mittels der Formel Länge x Breite x Höhe und erwerben die notwendige Rechenroutine. In der vorliegenden Stunde bringt die Lehrerin einen Kieselstein mit und lässt zunächst Ideen entwickeln, wie das Volumen des Kieselsteins ermittelt werden kann. Mehrere von den SchülerInnen eingebrachte Vorschläge verdichten sich zur ,Eintauchmethode $^{6}$ (vgl. Abb. 4).

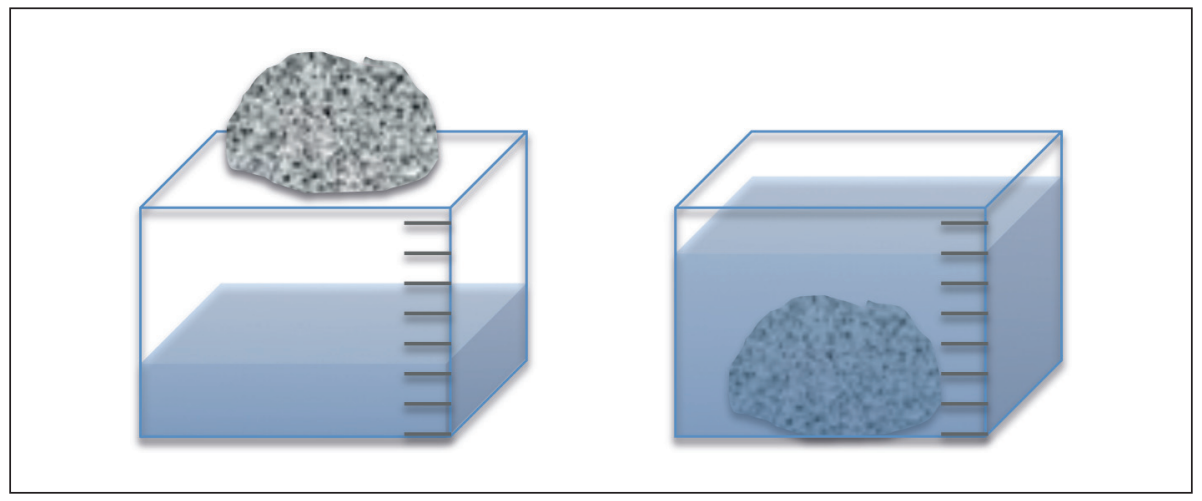

Abb. 4: Eintauchexperiment zur Volumenmessung (eigene Darstellung J. L.)

Die Lehrerin lässt das Experiment ohne Sprachhilfen durchführen, beschreiben und abschließend das Volumen berechnen. Das Schreibprodukt einer Partnergruppe (vgl. Abb. 5) zeigt eine alltagssprachliche Fassung, worin keine Fachbegriffe verwendet werden und fälschlicherweise das Steigen der Skala, und nicht das Steigen des Wasserspiegels, genannt wird. Mutmaßlich haben die SchülerInnen das Richtige gemeint, aber das Falsche geschrieben. 


\section{Schreibprodukt einer Gruppe ohne Sprachhilfen als Transkript:}

Im Quader sind, wenn man ibn bis zu $6 \mathrm{~cm}$ auf der Skala füllt, 300ml. Als wir den Stein binein getan haben ist die $S k$ ala auf $7,2 \mathrm{~cm}$ gestiegen.

Abb. 5: Schreibprodukt einer Partnergruppe

\section{Ausbildungsaufgabe:}

(1) Analysieren Sie das Schreibprodukt der SchülerInnen hinsichtlich der fachlichen und sprachlichen Stärken und Schwächen.

(2) Schreiben Sie den SchülerInnen eine Rückmeldung.

(3) Entwickeln Sie Sprachhilfen, mit welchen die SchülerInnen das Experiment strukturiert und sprachkompetent beschreiben können.

(4) Formulieren Sie fünf Empfehlungen für Lehrkräfte, die es bei der Standardsituation Versuchsbeschreibung zu berücksichtigen gilt.

\section{Lösungsvorschlag für die Teilaufgabe 3:}

\section{Wortliste}

- das (quaderförmige) Gefäß

- der (beliebig geformte) Stein

- der Quader

- das Volumen

- die Skala, die Messskala

- der Wasserspiegel

- die Differenz

- steigen um/auf

- sich vergrößern um

- ein/füllen in

- berechnen mit

\section{Wortfeld}

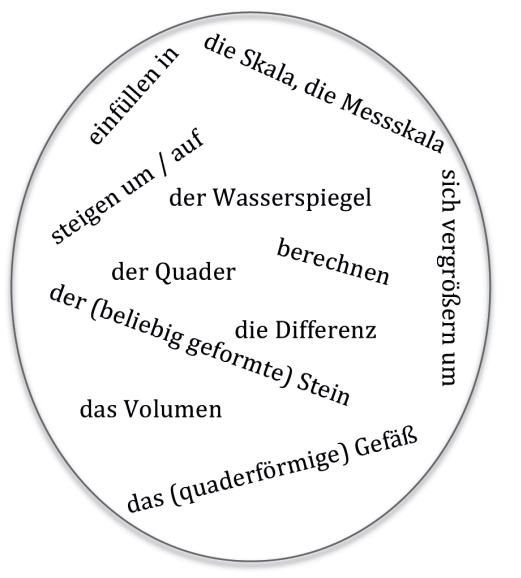

Abb. 6: Wortliste und Wortfeld (eigene Darstellung J. L.) 


\section{Leitfragen:}

- Was habe ich?

- Was mache ich?

- Was sehe ich?

- Was erkenne ich?

- Wie nutze ich dies?

Sachverhalte können in sehr verschiedenen Symbolisierungsformen repräsentiert bzw. dargestellt werden. Diese liegen auf verschiedenen Abstraktionsebenen (vgl. Abb. 7) und haben hinsichtlich des Verstehens und des Kompetenzerwerbs spezifische didaktische und methodische Funktionen. Die folgende Grafik zeigt den Weg durch die Landschaft der Darstellungsformen.

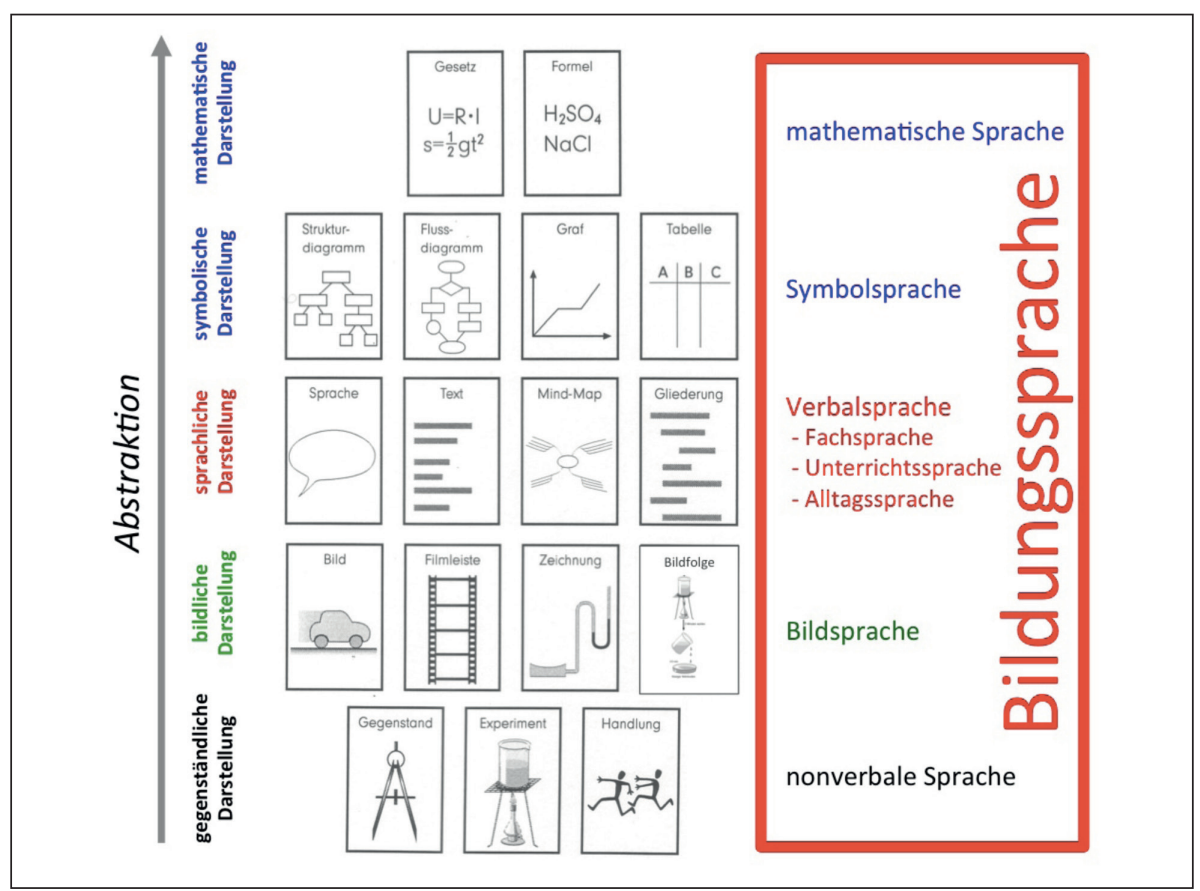

Abb. 7: Darstellungsformen auf Darstellungsebenen (eigene Darstellung J. L.) 
Die folgende Abbildung (Abb. 8) zeigt den vielfältigen Wechsel der Darstellungsformen und -ebenen in dem Beispiel zur Volumenberechnung.
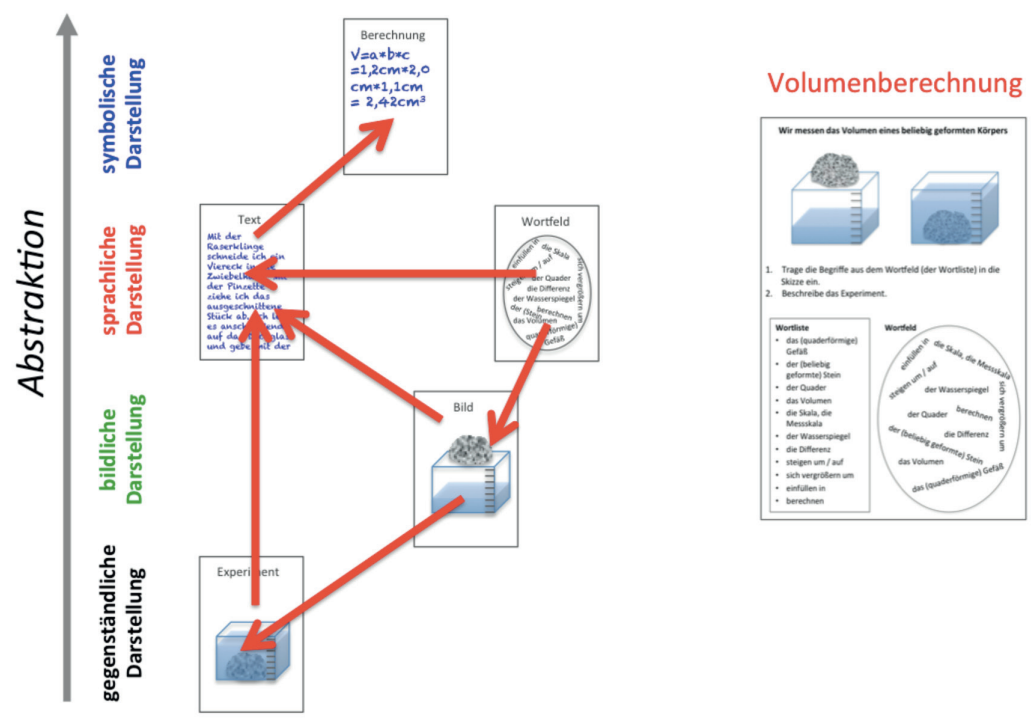

Abb. 8: Wechsel der Darstellungsformen (eigene Darstellung J. L.)

\subsection{Beispiel 2: Beschreibung des Druckmessers}

In der Klasse 8 soll der Druckmesser beschrieben werden. SchülerInnen neigen bei diesem Auftrag dazu, mit dem zu starten, was ins Auge fällt: „Da war rotes Wasser drin. Wenn man zu fest draufdrückt, dann spritzt das Wasser oben raus.“ Eine Gerätebeschreibung mit dem Augenfälligen - hier dem roten Wasser - zu starten ist für SchülerInnen naheliegend, jedoch in der Regel nicht passend. Das folgende Schreibprodukt (Abb. 9) einer Schülerin ist erfolgreich, wenngleich nicht fehlerfrei, weder fachlich noch sprachlich. 


\section{Schreibprodukt einer Schülerin als Transkript:}

\section{Hausaufgabe (Der Druckmesser)}

Der Druckemesser besteht aus einer Drucksonde, ein Manometer, eine Metallsonde, ein Membran, ein (Luft)Rohr, Wasser und einer Skala. Das Rohr ist mit der Metalldose, auf der die Membranen sind verbunden. In diesem Rohr befindet sich Wasser und Luft. Drückt man nun auf die Membranen wird die Luft im Rohr nach oben gedrückt. Dieser Druck bzw. die Luft drück.t dann auf das Wasser. Da das Rohr zwei Kurven um die Skala macht kann man sehen auf welchem Punk.t nun das Wasser steht.

Abb. 9: Schreibprodukt einer Schülerin

Abgesehen von der falschen Dativdeklination wird von Membranen gesprochen, obwohl es nur eine Membran gibt. Fachlich sollte unter Einbindung der gesamten Klasse hier die interessante Frage geklärt werden, ob der Druck oder die Luft auf das Wasser, drückt'. An diesem Lernprodukt lassen sich sprachliche und fachliche Fehler deutlich voneinander trennen und die erfolgreiche Bearbeitung schafft Gelegenheiten zu Anschlusskommunikation über Fachliches und Sprachliches. Eine gute Aufbaubeschreibung setzt die Kenntnis der ,Architektur ${ }^{6}$ des Objektes voraus. Diese zu erkennen ist für SchülerInnen schwierig oder gar unmöglich. Deshalb benötigen sie strukturelle Hilfen.

\section{Ausbildungsaufgabe:}

(1) Analysieren Sie das Schreibprodukt der Schülerin hinsichtlich der fachlichen und sprachlichen Stärken und Schwächen.

(2) Schreiben Sie der Schülerin eine Rückmeldung.

(3) Entwickeln Sie ein Arbeitsblatt mit Methoden-Werkzeugen, so dass die SchülerInnen ein erfolgreiches Schreibprodukt zur Beschreibung der Druckdose erstellen können.

(4) Begründen Sie Ihre Entscheidungen unter Rekurs auf die Prinzipien des Sprachlernens im Fach und auf die Merkmale guter Aufgabenstellungen. 
(5) Formulieren Sie fünf Empfehlungen für Lehrkräfte, die es bei der Standardsituation Versuchsbeschreibung zu berücksichtigen gilt.

\section{Lösungsvorschlag für die Teilaufgabe 3:}

Das nachfolgende Arbeitsblatt enthält drei Methoden-Werkzeuge. Die beschriftete Skizze stellt die Fachbegriffe mit Artikel zur Verfügung. Die Formulierungshilfen dienen der sprachlichen Unterstützung, während das Strukturdiagramm die Architektur des Gerätes darstellt und den Schreibprozess logisch-strukturell unterstützt.

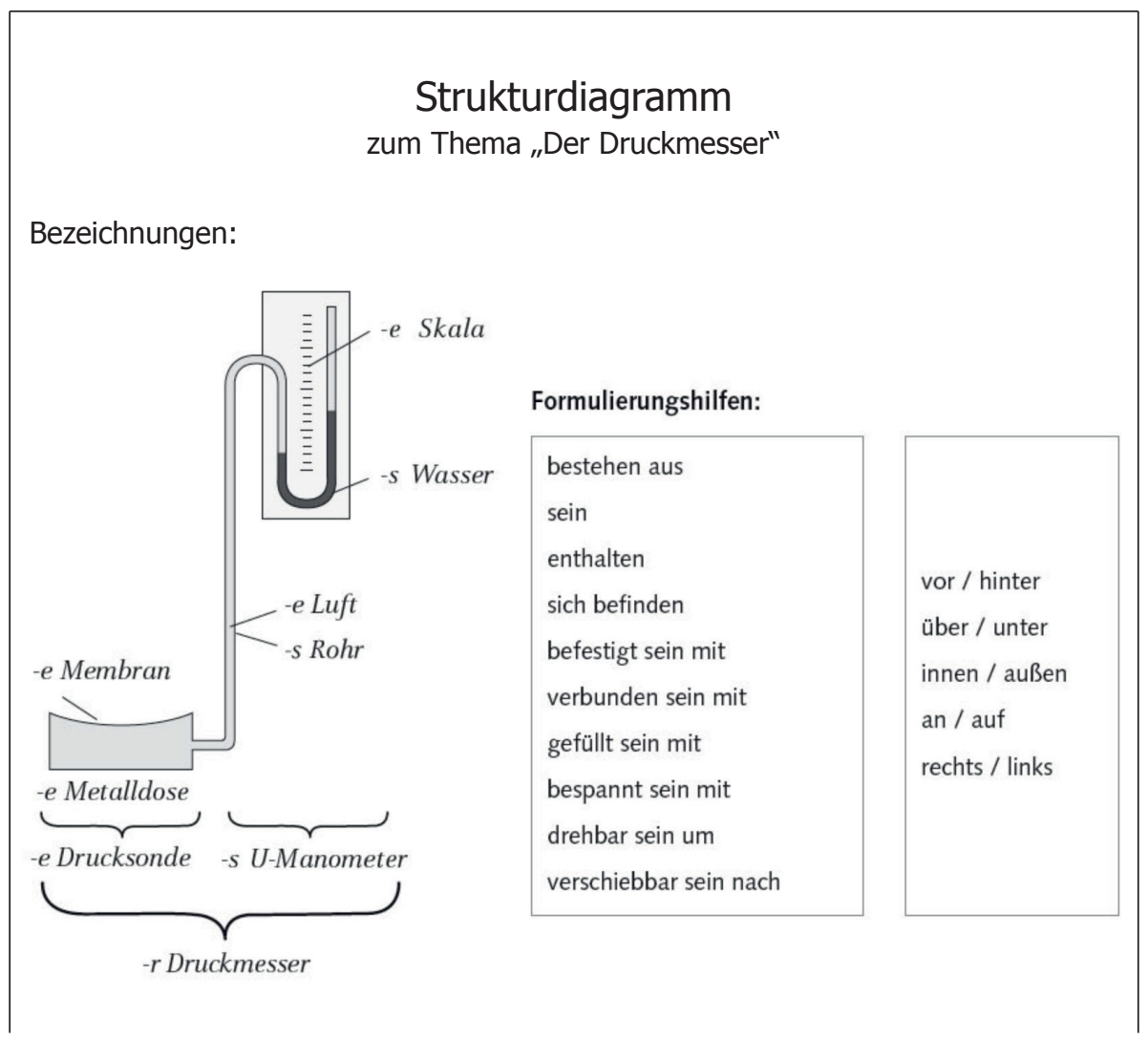




\section{Aufgaben:}

1. Schreibe die Verben an die Pfeile des Diagramms.

2. Beschreibe die Druckdose mit Hilfe des Strukturdiagramms.

Strukturdiagramm:

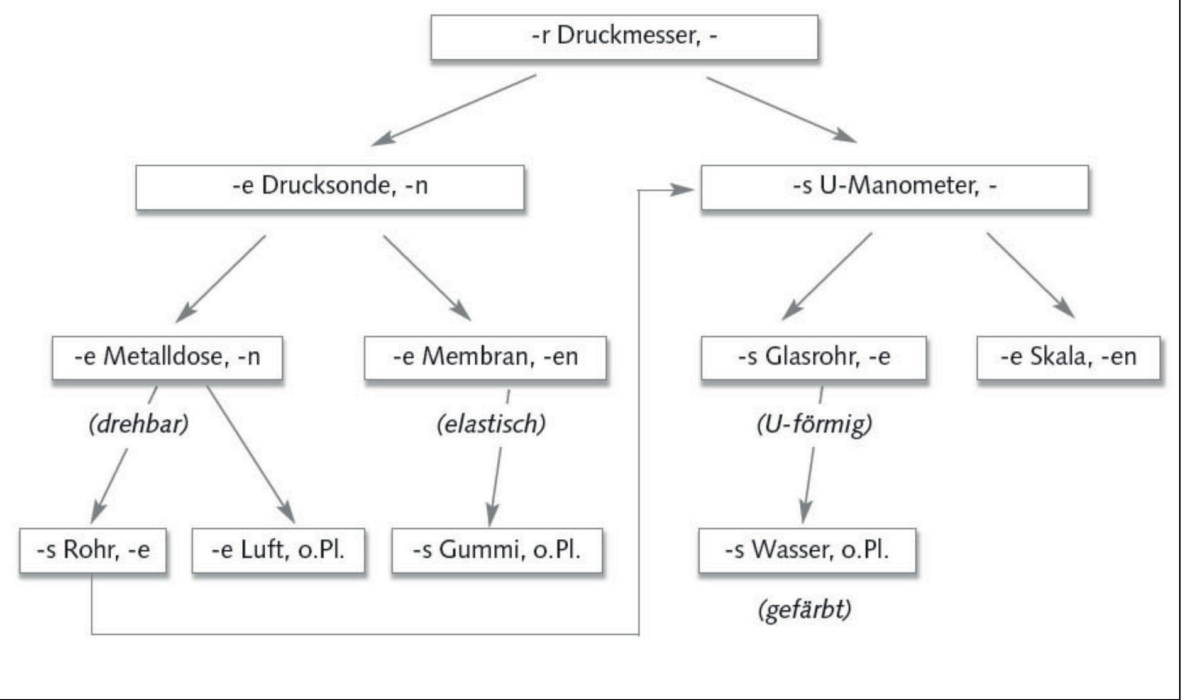

Abb. 10: Arbeitsblatt (Leisen 2013: 47)

Sprachliche Unterstützung darf nicht zu einer Absenkung des Anforderungsniveaus führen. Das angemessene Niveau kann variantenreich über Darstellungsformen eingestellt werden. Diese liegen auf verschiedenen Abstraktionsebenen und haben hinsichtlich des Verstehens und des Kompetenzerwerbs spezifische didaktische und methodische Funktionen. Die folgende Grafik zeigt den Weg durch die Landschaft der Darstellungsformen. 


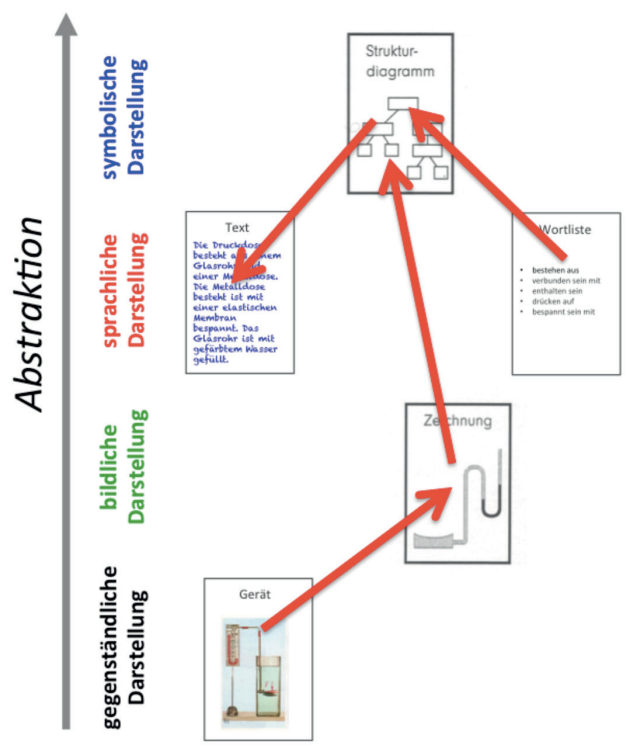

Druckdose

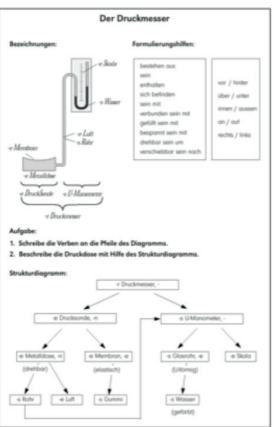

Abb. 11: Wechsel der Darstellungsformen (eigene Darstellung J. L.)

\section{Ausbildungsaufgaben, Vorhaben und Ausbildungsmodule als Elemente in der Sprachbildung}

\subsection{Ausbildungsaufgaben}

Ausbildungsaufgaben können in verschiedenen Ausbildungsformaten und -veranstaltungen eingesetzt werden. Ihr Hauptzweck besteht darin, die Studierenden bzw. die ReferendarInnen in die eigentätige Auseinandersetzung mit der Thematik zu bringen. Ausbildungsaufgaben können in Seminarsitzungen und in Vorhaben eingesetzt werden. Gutes Lernen braucht immer Wissensinput, Eigentätigkeit und Austausch. Eine Seminarsitzung sollte unbedingt entsprechende Phasen enthalten, die durchaus je nach Umfang mehrfach auftreten. 
- Wissensinput: Dieser kann erfolgen über einen Vortrag, einen Informationsbaustein, z.B. Handout, ein Kompendium, eine E-Learning-Einheit, ein Skript. Der Wissensinput kann vorbereitend erfolgen oder in die Sitzung integriert sein.

- Eigentätigkeit: Die Eigentätigkeit wird eingeleitet durch entsprechende Aufgabenstellungen. Es empfiehlt sich eine Partner- oder Kleingruppenarbeit. Das Ergebnis ist ein sogenanntes Lernprodukt, z.B. eine Analyse von sprachsensiblem Material, Vorschläge sprachsensibler Aufgabenstellungen, sprachsensibel gestaltete Lerntexte, Analyse von Sprachproblemen an einem Videotranskript.

- Plenumsdiskussion: Eine Plenumsdiskussion kann sich sehr ergiebig an präsentierten Lernprodukten entfalten. Damit wird der Mehrwert, der in den verschiedenen Lernprodukten liegt, genutzt.

Ein praxisorientiertes Ausbildungskonzept muss

- von konkreten sprachlichen Handlungssituationen zur Sprachbildung im Sach-Fachunterricht und von Fragenstellungen zur Sprachbildung ausgehen.

- anhand konkreter Beispiele, Materialien, Modelle, Lernprodukte etc. modellhaft sprachsensibles Handeln im Sach-Fachunterricht zeigen.

- theoriegestützt und theoriebegleitet das unterrichtliche Handeln zur Sprachbildung auf der Hintergrundfolie der Spracherwerbstheorie und der Sprachdidaktik hinterfragen.

- in Schleifen und in mehreren Durchläufen Wissen wiederholen, vertiefen und aus neuer Perspektive angehen. 
Letztlich muss Wissen professionell in Handlungssituationen genutzt werden, was ein langwieriger und auch mühsamer Prozess ist, der Zeit benötigt. Die Ausbildung zu einem sprachsensiblen Sach-Fachunterricht ist wirksam und nachhaltig, wenn die Studierenden

- sich über einen längeren Zeitraum in Schleifen gestuft mit dem Thema beschäftigen,

- an wechselnden Aufgabenstellungen gemeinsam intensive Arbeitskontakte haben,

- an guten Modellen und Beispielen fallorientiert lernen,

- in passendem Umfang Input und theoretisches Hintergrundwissen erhalten,

- sich individuell mit der Sache auseinandersetzen,

- sich mit anderen diskursiv austauschen,

- ihr Wissen und ihre Erfahrungen einbringen und ausbauen

- und dabei betreut werden,

- ihr Könnensbewusstsein stärken.

\subsection{Vorhaben}

Seminarsitzungen in wöchentlicher Folge sind zeitlich begrenzt. Eine professionell nachhaltige Ausbildung erfordert ein in Schleifen angelegtes Lernen mit Elementen aus und in der Praxis. Hier ist das Vorhaben ein geeignetes Format. Ein Vorhaben ist eine über einen längeren Zeitraum geplante Unternehmung mit einer klaren Zielsetzung - hier Professionalisierung im Bereich der Sprachbildung - und entsprechenden Maßnahmen zur Zielerreichung. Ein Vorhaben muss zeitlich und thematisch begrenzt sein, muss eine klare zeitliche Ablaufstruktur haben und das Arbeitsprodukt muss definiert sein. Gruppen von drei bis vier Studierenden wählen ein Vorhaben aus, das sie über drei Monate (ein Semester) hinweg bearbeiten. Die folgende Tabelle (Tab. 1) listet mögliche Vorhaben aus vier verschiedenen Bereichen der Sprachbildung auf. 
Tab. 1: Vorhaben in der Sprachbildung

\begin{tabular}{|c|c|}
\hline $\begin{array}{l}\text { 1. Herkömmliche Aufgabenstellungen } \\
\text { sprachsensibel ausbauen } \\
\text { 2. Wechsel der Darstellungsformen } \\
\text { sprachbildend einsetzen } \\
\text { 3 Das Begriffslernen und den (Fach) } \\
\text { Wortschatz gezielt fördern }\end{array}$ & $\begin{array}{l}\text { Bildungssprache } \\
\text { gezielt fördern }\end{array}$ \\
\hline $\begin{array}{l}\text { 4. An Transkripten, Videoszenen, Sprach- } \\
\text { produkten Sprachdiagnose betreiben } \\
\text { 5. Bildungssprachliche Merkmale analysie- } \\
\text { ren, spezifische Sprachprobleme untersu- } \\
\text { chen und Hilfen entwickeln } \\
\text { 6. Binnendifferenzierende Methoden-Werk- } \\
\text { zeuge, gestufte Sprachhilfen, Methoden } \\
\text { des Scaffolding entwickeln/einsetzen }\end{array}$ & $\begin{array}{c}\text { Sprachprobleme diagnostizieren } \\
\text { und Sprachhilfen einsetzen }\end{array}$ \\
\hline $\begin{array}{l}\text { 7. Lehrtexte sprachsensibel vereinfachen } \\
\text { und umgestalten } \\
\text { 8. Fachtexte mit Lesehilfen und } \\
\text { Lesestrategien aufbereiten } \\
\text { 9. Das Schreiben im Sach-Fachunterricht } \\
\text { mit Methoden-Werkzeugen unterstützen }\end{array}$ & $\begin{array}{l}\text { Lese- und Schreibkompetenzen } \\
\text { fördern }\end{array}$ \\
\hline $\begin{array}{l}\text { 10. Sprachübungen für fachspezifische } \\
\text { Sprachsituationen konzipieren } \\
\text { 11. Leseübungen zu Fachtexten konzipieren } \\
\text { 12. Schreibübungen konzipieren }\end{array}$ & Bildungssprache üben \\
\hline
\end{tabular}

Eine Aufgabenstellung für ein Vorhaben könnte lauten:

Aufgabenstellung: Bilden Sie eine Kleingruppe von drei bis vier Studierenden.

(1) Wählen Sie ein Vorhaben aus und entwerfen Sie eine Lernumgebung im Sinne des sprachsensiblen Sach-Fachunterrichts (Zeitraum: vier Wochen).

(2) Stellen Sie das Vorhaben und die geplante Lernumgebung nach Terminabsprache vor und lassen sich von dem/der AusbilderIn beraten. 
(3) Unterrichten Sie (sofern möglich) eine geeignete Lerngruppe und evaluieren Sie den Unterricht anhand der Lernprodukte (Zeitraum: vier Wochen).

(4) Reflektieren Sie die Konsequenzen für Ihre weitere berufliche Arbeit (Zeitraum: zwei Wochen).

(5) Präsentieren Sie in der Abschlusssitzung ausgewählte Ergebnisse (Zeitraum: zwei Wochen).

\subsection{Ausbildungsmodule}

Ausbildungsmodule sind zeitlich umfangreichere Ausbildungsformate mit einer zeitlichen Abfolge von Seminarsitzungen und Arbeitsphasen. Vorhaben sind ein Ausbildungselement innerhalb eines Ausbildungsmoduls mit Wissensinput und Ausbildungsaufgaben.

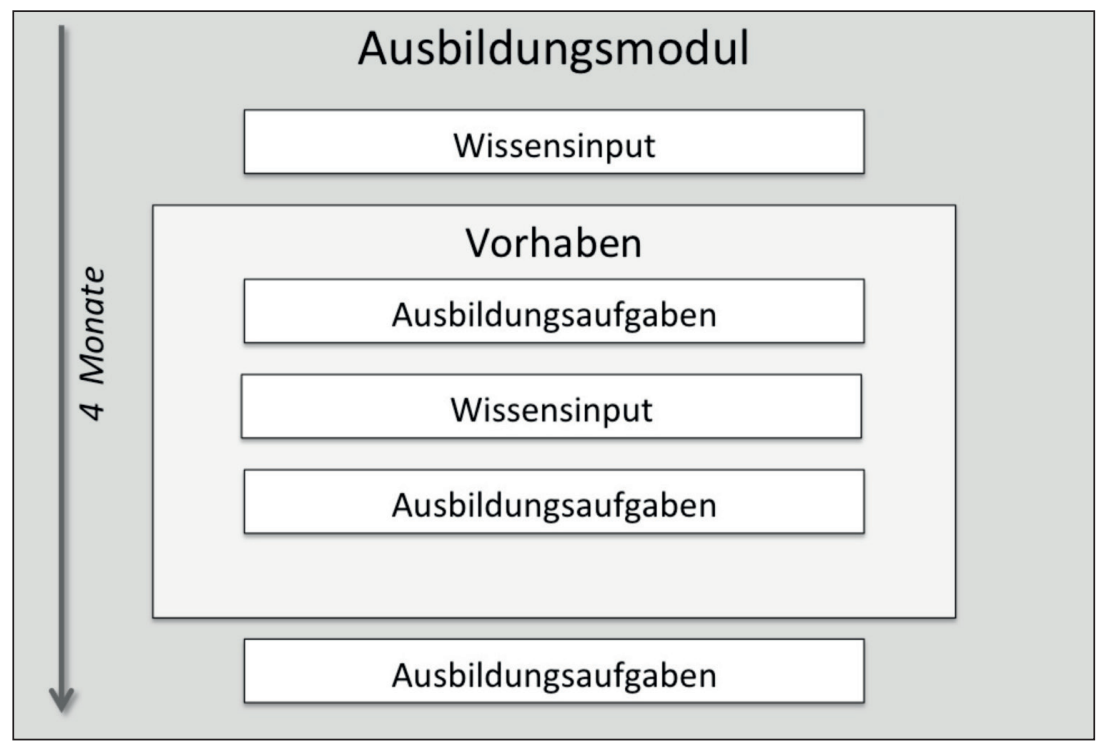

Abb. 12: Ausbildungsmodule - Vorhaben - Ausbildungsaufgaben (eigene Darstellung J. L.) 
Die folgende Tabelle (Tab. 2) zeigt eine mögliche Zeitstruktur eines Ausbildungsmoduls. Die praxisbezogenen Arbeitsphasen werden von dem/der AusbilderIn beraten und betreut.

Tab. 2: Zeitstruktur eines Moduls

\begin{tabular}{|c|c|}
\hline Phase & Themen - Inhalte - Aufgaben \\
\hline 1. Sitzung & $\begin{array}{l}\text { - Einführung in die Thematik (Vortrag- } \\
\text { Ausbildungsaufgaben - Diskussion) } \\
\text { - Vorstellung von Vorhaben }\end{array}$ \\
\hline $\begin{array}{l}\text { 1. Arbeitsphase } \\
\text { (4 Wochen) }\end{array}$ & $\begin{array}{l}\text { - Diagnose von Sprachprodukten im eigenen } \\
\text { Unterricht } \\
\text { - Analyse und Reflexion der Sprachprodukte } \\
\text { - Aufgabenerstellung von Sprachförderung }\end{array}$ \\
\hline 2. Sitzung & $\begin{array}{l}\text { - Vorstellung und Diskussion der } \\
\text { Sprachprodukte } \\
\text { - Erfahrungsaustausch - Beratung } \\
\text { - Weiterer Input }\end{array}$ \\
\hline $\begin{array}{l}\text { 2. Arbeitsphase } \\
\text { (4 Wochen) }\end{array}$ & $\begin{array}{l}\text { - Einsatz der Sprachförderaufgaben } \\
\text { - Analyse und Reflexion der Wirksamkeit }\end{array}$ \\
\hline 3. Sitzung & $\begin{array}{l}\text { - Abschlusspräsentation und Auswertung } \\
\text { - Erfahrungsaustausch - Anregungen zur } \\
\text { Weiterarbeit }\end{array}$ \\
\hline
\end{tabular}

\section{Zusammenfassung}

Die Ausbildung für die Sprachbildung im sprachsensiblen Sach-Fachunterricht ist eine Querschnittsaufgabe der Fachdidaktik der Fächer, der Sprachlerndidaktik und der Fremdsprachendidaktik (vgl. Abb. 2). Somit ist das Thema universitär mehrfach beheimatet, was jedoch die Gefahr birgt, dass sich keine Institution 
zur Gänze verantwortlich fühlt. Gemeinsame Standards, Absprachen und Kooperationen sind die Voraussetzung für eine gelingende Ausbildung, stellen aber noch kein Ausbildungsmodell dar. Dieses muss vor Ort durch Lehrpersonen in Zusammenarbeit mit Studierenden in geeigneten Formaten gestaltet werden. Ausbildungsaufgaben sind dafür eine geeignete Umsetzungsmöglichkeit. Eine Ausbildungsaufgabe ist - wie oben ausgeführt - eine dem Lernraum entnommene Aufgabe zur beruflichen Kompetenzentwicklung (hier sprachsensibler Sach-Fachunterricht) der Studierenden. Sie enthält Materialien aus der Praxis der Sprachbildung (Lehr- und Lernmaterialien, Lese- und Lernprodukte der LernerInnen etc.) und aus dem Bereich der Sprach- und Spracherwerbstheorie (theoretisches Hintergrundwissen). Studierende erstellen dabei auswertbare Lernprodukte, anhand derer ihr individueller Kompetenzstand rückgemeldet werden kann. Ausbildungsaufgaben ermöglichen es darüber hinaus, theoretisches Wissen mit der Unterrichtspraxis zu verknüpfen und unterrichtspraktische Erfahrungen zu sammeln, die - in komplexere Vorhaben eingebunden - im Sinne reflektierter Praxis an Wissen rückgebunden werden können.

\section{Literatur}

Baumann, B. \& Becker-Mrotzek, M. (2014). Sprachförderung und Deutsch als Zweitsprachen an deutschen Schulen: Was leistet die Lehrerbildung? Köln: Mercator-Institut für Sprachförderung und Deutsch als Zweitsprache.

Bund-Länder-Kommission für Bildungsplanung und Forschungsförderung (1997). Gutacbten ₹ur Vorbereitung des Programms „Steigerung der Effizienz des mathematisch-naturwissenschaftlichen Unterrichts". Materialien zur Bildungsplanung und Forschungsförderung Heft 60. Verfügbar unter: http://www.blk-bonn.de/papers/heft60.pdf [29.03.2016].

Cinar, M., Otremba, K., Stürzer, M. \& Bruhns, K. (2013). Kinder-Migrationsreport. Ein Datenund Forschungsüberblick zu Lebenslagen und Lebenswelten von Kindern mit Migrationshintergrund. München: Deutsches Jugendinstitut.

Deutsches Institut für Internationale Pädagogische Forschung (DIPF) (2006): Unterricht und Kompetenzerwerb in Deutsch und Englisch. Zentrale Befunde der Studie Deutsch-Englisch-SchülerleistungenInternational (DESI). Frankfurt: DIPF. 
Ellis, R. (2003). Task-based Language Learning and Teaching. Oxford, New York: Oxford Applied Linguistics.

Haug, S. (2008). Sprachliche Integration von Migranten in Deutschland. Working Paper 14 der Forschungsgruppe des Bundesamtes. Bonn: Bundesamt für Migration und Flüchtlinge.

Humboldt-Universität zu Berlin (2015): Studien- und Prüfungsordnung für die Studienanteile Bildungswissenschaften und Sprachbildung im Bachelorstudiengang mit Lehramtsoption bzw. Lehramtsbezug. Amtliches Mitteilungsblatt der Humboldt-Universität zu Berlin Nr. 80/2015.

Jürgens, E. (2016): Erfolgreich durch das Praxissemester - Gestaltung, Durchfübrung, Reflexion. Berlin: Cornelsen.

Klieme, E. et al. (2003): Zur Entwicklung nationaler Bildungsstandards. Eine Expertise. Bonn: Bundesministerium für Bildung und Forschung.

Kross, A. \& Lind, G. (2001). Lernen mit Beispielaufgaben in Biologie und Physik. Der mathematische und naturwissenschaftliche Unterricht, 8, 491-496.

Leisen, J. (2005). Zur Arbeit mit Bildungsstandards - Lernaufgaben als Einstieg und Schlüssel. Der mathematische und naturwissenschaftliche Unterricht, 58 (5), 306-308.

Leisen, J. (2011). Aufgabenstellungen in Schule und Ausbildung. SEMINAR, 4, 57-74.

Leisen, J. (2013). Handbuch Sprachförderung im Fach - Sprachsensibler Sach-Fachunterricht in der Praxis. Stuttgart: Klett.

Lütke, B. (2010). Deutsch-als-Zweitsprache in der universitären Lehrerausbildung. Der fachintegrative Ansatz im Master of Education an der Humboldt- Universität zu Berlin. In B. Ahrenholz (Hrsg.), Fachunterricht und Deutsch als Zweitsprache (153-166). Tübingen: Narr.

Stäudel L., Bohl, T., Merk, S. \& Rehm, M. (2012). Aufgaben im naturwissenschaftlichen Unterricht. Allgemeindidaktische, fachdidaktische und fachliche Expertise. journal für lehrerinnen- und lebrerbildung, 1, 26-33.

Trägerkonsortium BISS (2012). Expertise „Bildung durch Sprache und Schrift (BISS)“. (BundLänder-Initiative zur Sprachförderung, Sprachdiagnostik und Leseförderung). Verfügbar unter: https://www.bmbf.de/files/BISS_Expertise.pdf [29.03.2016].

Weinert, F. E. (2001). Leistungsmessung in Schulen - Eine umstrittene Selbstverständlichkeit. In F. E. Weinert (Hrsg.), Leistungsmessung in Schulen. Weinheim, Basel: Beltz.

Barbara Hinger (Hg.), Zweite „Tagung der Fachdidaktik“ 2015.

Sprachsensibler Sach-Fach-Unterricht - Sprachen im Sprachunterricht.

(C) 2016 innsbruck university press, ISBN 978-3-903122-51-2, DOI 10.15203/3122-51-2 